\title{
Estimating the Maximum Intensities of Soft X-ray Flares Using Extreme Value Theory
}

\author{
V. De la $\mathrm{Luz}^{1}$ - E.P. Balanzario ${ }^{2}$ - T. Tsiftsi ${ }^{2}$

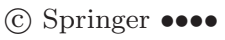

\begin{abstract}
Solar flares are one of the most energetic events in the solar system, their impact on Earth at ground level and its atmosphere remains under study. The repercussions of this phenomenon in our technological infrastructure includes radio blackouts and errors in geopositional and navigation systems that are considered natural hazards in ever more countries. Occurrence frequency and intensity of the most energetic solar flares are been taken into account in national programs for civil protection in order to reduce the risk and increase the resilience from Space Weather events. In this work we use the statistical theory of extreme values as well as other statistical methods in order to asses the magnitudes of the most extreme solar flare events expected to occur in a given period of time. We found that the data set under study presents a dual tail behaviour. Our results show that on average we can expect one solar flare greater than X23 each 25 years, that is to say, one such event each two solar cycles.
\end{abstract}

Keywords: Flares, Forecasting; Solar Cycle, Observations; Spectrum, X-Ray

\section{Introduction}

One of the most energetic stellar activities is produced by flares (Katsova et al., 2018). In the case of the Sun the flares occur over the solar surface, mainly in active regions (Toriumi et al., 2017). In these areas, the magnetic structures can produce very large amounts of energy that are released in the magnetic reconnection process (Benz and Güdel, 2010). This magnetic reconnection has the ability to perform a transformation of energy between magnetic and kinetic energy. The full process that involves solar reconnection and its changes in energy

\footnotetext{
1 Conacyt - SCiESMEX - LANCE, Instituto de Geofísica, Unidad Michoacán, Universidad Nacional Autónoma de México, Morelia, Michoacán, 58190, México. email: vdelaluz@igeofisica.unam.mx

2 Centro de Ciencias Matemáticas, Universidad Nacional Autónoma de México, Apartado Postal 61-3 (Xangari), Morelia Michoacán, México.
} 
distribution of the system's surroundings is known as a solar flare (Gold and Hoyle, 1960).

When the charged particles are accelerated by a flare, they can produce an amount of electromagnetic waves at all wavelengths of the spectrum (in the most energetic, even gamma rays (Ackermann et al., 2014)). Their intensity and dynamic evolution depends on their interactions with their environments (Canfield et al., 1993). The emission at soft X-ray wavelengths produced during a solar flare has been used as a measure of the intensity of the whole complex solar flare process (Chertok and Belov, 2017). The X-ray flare classification shows indirectly the amount of energy released during a solar flare event (Chamberlin et al., 2009). The statistical study of occurrences of solar flares using X-ray classification allows us to estimate the magnitudes of the most extreme intensities a solar flare can reach in a given period of time (Koons, 2001; Riley, 2012). Knowing the magnitude of the most extreme events is of great interest in the context of Space Weather studies due to the fact that the protocols of civil protection related with this natural phenomenon need information regarding the worst expected scenarios (Jonas and McCarron, 2016).

The WSPC/NOAA has regular records of solar flares since 1975 until the present day. From 1975 up to 1978 the records used $\mathrm{H} \alpha$ observations, but after 1978 a set of GOES X-ray detectors were used in order to record the solar flare events. SWPC has made efforts in order to maintain the X-ray solar flare classification constant in between changes of detectors installed in the space crafts and they released an homogeneous public list of solar flare records from 1 September 1975 up to 28 June 201柯.

In this work we use the methods of the statistical theory of extreme values (see Castillo et al. (2005); Coles (2001); de Haan and Ferreira (2007)) as well as ad hock statistical methods in order to asses the magnitudes of the most extreme solar flare events that can be expected to occur in the following years. For this purpose we use the NOAA X-ray classification data set from 5 November 1975 to 9 October 2017.

In Section 2 we set up the statistical problem. Section 3 describes the raw data. In Section 4 we study the tail behavior of the distribution of the solar flare intensities. We do this by fitting five different models for the distribution under study.

\section{Setting up the statistical problem}

We are interested in estimating the probability of a solar flare intensity exceeding a threshold $x$. For this end, we need information about the upper tail of the distribution of values $X$ of a solar flare intensity. Denote by $X_{(n)}$ the maximum of a sample $X_{1}, \cdots, X_{n}$ of size $n$ of the random variable $X$ and write

$$
p_{n}(x)=\mathrm{P}\left\{X_{(n)}>x\right\} .
$$

\footnotetext{
${ }^{1}$ The data can be found in https://www.ngdc.noaa.gov/stp/space-weather/solar-data/
} solar-features/solar-flares/x-rays/goes/xrs/ 
By the tail of the distribution of $X_{(n)}$ we mean the behavior of $p_{n}(x)$ as $x \rightarrow \infty$. For a fixed $n$, the slower $p_{n}(x)$ tends to zero as $x \rightarrow \infty$, the greater the probability of observing the event $\left\{X_{(n)}>x\right\}$. An inspection of the raw data to be depicted shortly leads us to expect a tail behavior of the form

$$
p_{n}(x) \propto x^{-\alpha}
$$

for a suitable positive $\alpha$ to be estimated from the data set. Since $X$ and $X_{(n)}$ belong to the same domain of attraction of a max-stable distribution, then the value of $\alpha$ does not depend on $n \in \mathbb{N}$ (see (Leadbetter, Lindgren, and Rootzén, 2012), page 15). Notice also that the smaller the value of $\alpha$, the slower $p_{n}(x)$ tends to zero as $x \rightarrow \infty$. Therefore, the smaller the value of $\alpha$, the larger the probability that $X_{(n)}$ assumes a large value.

By using the collected data set corresponding for the intensities $X$ of the observed solar flares, we approach the task of estimating the parameter $\alpha$ which determines the tail behavior of the distribution of $X_{(n)}$ by each of the following methods.

1. By fitting a log-logistic model to small random subsets of the whole data set.

2. By the block approach of the extreme value theory, where we fit a Fréchet distribution to the maxima $X_{(n)}$ of blocks of size $n$ from the data set (Coles (2001), chapter 3).

3. By the threshold approach of the extreme value theory, where we fit a Pareto distribution to the excesses $X_{(n)}-u$ for a given $n$ and a threshold value $u$ (Coles (2001), chapter 4).

4. By fitting to the entire data set a probability distribution composed of a $\log$-logistic part and a Pareto part.

5. By fitting to the entire data set a probability distribution composed of a log-logistic part and a tempered Pareto part.

It will turn out that each of these methods of estimating $\alpha$ have advantages and disadvantages. By comparing the estimates for $\alpha$ obtained by each of the above methods, we are in a position to offer a wide perspective from which one can gain an informed understanding of the likelihood of observing an extremely large solar flare.

\section{Description of the raw data}

In Figure 1 we can see a graphical representation the magnitude (in $\mathrm{W} \mathrm{m}^{-2}$ ) of the observed solar flare intensities at a given time from 5 November 1975 to 9 October 2017. The data set here analyzed consists of 77370 data points. In columns with labels "Range" and "Data" of Table 1 we further describe the distribution of the solar flare intensities. We notice that an overwhelming majority of the intensities are not greater than $0.0001\left(\mathrm{~W} \mathrm{~m}^{-2}\right)$, this peak flux value represents an X1 GOES-class flare. Only a relatively few observations $(0.64 \%)$ lie above the value $0.0001\left(\mathrm{~W} \mathrm{~m}^{-2}\right)$. On the other hand, it is apparent that these extreme observations are much greater than $0.0001\left(\mathrm{~W} \mathrm{~m}^{-2}\right)$. Having 
few observations much greater than the body of the data points suggests a behavior for the tail distribution as depicted in Equation 1 with a positive $\alpha$. The maximum observed value equals $0.0028\left(\mathrm{~W} \mathrm{~m}^{-2}\right)$. See Table 2] where we report a list of the intensities of the most severe events in our data set together with their dates of occurrence.

The gray line in Figure 1 is proportional to the density $d(t)$ of the number of events per unit time. Notice in particular that this density is not uniform over time. The maximum value for the density $d(t)$ occurs at $t=1982.08$ and this maximum equals 0.045 , which represents 9.59 events per day. The minimum of $d(t)$ occurs at $t=2008.7$ and this minimum equals 0.0058 , which corresponds to 1.23 events per day. The average number of events over the whole period of time here considered equals 5.05248 flares per day.

We computed the autocorrelation function for the raw data for distinct lags. Small values for the autocorrelations for positive lags were found, in spite of the fact that large values for solar flare intensities tend to occur in clusters (see for example, entries 12 to 15 in Table 2). The small values for the autocorrelations suggest, at least in a first approximation, that the data set comes form independent observations of the random variable $X$. Having a data set consisting of independent observations of a random variable allows us to apply with confidence distinct results from the theory of probability and statistics, for example, the procedures for the estimation of the parameters of a proposed model.

\section{Tail behavior}

In the second column of Table 3 we report the estimates for the tail index $\alpha$, defined by Equation 1, as given by the models considered in this work. In the third column of the table, we report 95\% confidence intervals for $\alpha$. In each case, the estimation for $\alpha$ and the confidence interval were computed from a sample of size given in the fourth column of Table 3. In the next subsections we comment some details about the estimation procedures reported in Table 3 .

\subsection{Log-logistic model}

Among the five models considered, the log-logistic is the most simple minded, but even if it only provides us a rather naive way to estimate the probabilities of large solar flare intensities, the log-logistic model provides us a rough model for the phenomenon we are interested in. Now, if we model a solar flare intensity $X$ by a log-logistic distribution, then we have

$$
\mathrm{P}\{X>x\}=\frac{1}{1+(x / \sigma)^{\alpha}} \sim\left(\frac{x}{\sigma}\right)^{-\alpha}
$$

when $x$ is large, and where the first equality in Equation 2 defines the loglogistic distribution. When fitting the log-logistic distribution to our data set, the parameters $\alpha$ and $\sigma$ in Equation 2 were estimated by the maximum likelihood method. However, for the log-logistic model we performed the estimation of parameters, not from the full data set consisting in 77370 points, but from a 
smaller random sub-sample of size 500, as reported in Table 2 For the null hypothesis stating that the chosen sub-sample of size 500 comes from a log-logistic distribution, the Pearson chi squared statistic for the evaluation of the goodness of fit has a $p$-value of 0.066 . Thus, the null hypothesis is not rejected, but one should remark that the $p$-value decreases quickly to zero when the sub-sample size increases. By applying both, the method of normal approximation and the bootstrap method, we found that $(1.25,1.45)$ is a $95 \%$ confidence interval for $\alpha$. When we substitute the estimated values $\hat{\alpha}=1.3492$ and $\hat{\sigma}=1.6240 \times 10^{-6}$, we obtain from Equation 2 that

$$
\mathrm{P}\{X>x\} \sim \frac{1.5439 \times 10^{-8}}{x^{1.3492}}
$$

for large values of $x$. We will come back to the log-logistic model when we analyze our data set by plotting it on log-log scale.

\subsection{The block approach}

Here we investigate the distribution of $X_{(n)}=\max \left\{X_{1}, \cdots, X_{n}\right\}$ for a given value of $n$, where $n$ is the size of a block in the block approach. In view of the estimated value for $\alpha$ obtained for the log-logistic model, then according to the Tippet-Fisher theorem from the extreme value theory, we may expect that $X_{(n)}$ has a Fréchet distribution (de Haan and Ferreira (2007), page 6). Equivalently, we expect that $X_{(n)}$ has a distribution given by

$$
\mathrm{G}_{\mathrm{ev}}(x)=\exp \left\{-\left[1+\xi\left(\frac{x-\mu}{\sigma}\right)\right]^{-1 / \xi}\right\}
$$

with a positive value for the shape parameter $\xi$.

Assuming that the events are distributed uniformly over time (with a rate of 5.05248 events per day), then a block size of $n=1300$ corresponds to approximately 257 days of solar activity. This gives us a sample of maximum values of size 59 from which we can estimate the parameter $\alpha$, which in the model given by Equation 4 is given by $\alpha=1 / \xi$.

With the above value for $n$, the parameters $\mu, \sigma$ and $\xi$ where estimated by the maximum likelihood method. Using this method we found that $\hat{\mu}=$ $3.313 \times 10^{-4}, \hat{\sigma}=2.854 \times 10^{-4}$ and $\hat{\xi}=0.4145$. From this estimate for $\xi$ we obtain that $\hat{\alpha}=2.4122$. By using the method of normal approximation, we obtained a $95 \%$ confidence interval for $\xi$ given by $(0.179,0.650)$. From this we can obtain a confidence interval for $\alpha$, as reported in Table 3. For the null hypothesis stating that the 59 maximum values of blocks of size 1300 come from a Fréchet distribution (equivalently, from the distribution given by Equation 4) the Pearson chi squared statistic has a $p$-value of 0.918 . Thus, we have a good fit of the maxima to the estimated model.

2 See DiCiccio and Efron (1996) and Diaconis and Efron (1983) for a description of the bootstrap method. 


\subsection{The threshold approach}

In order to apply the threshold approach to the estimation of $\alpha$, it is necessary to form a set of excesses $X_{(n)}-u$ over a threshold $u$. In order for this approach to make sense in the study of the intensities of solar flares, it is necessary that $X_{(n)}$ has a distribution with a tail similar to a power law tail, as depicted in Equation 11 In this case, it is expected that the excesses $X_{(n)}-u$ has a Pareto distribution defined by

$$
H(x)=1-\left(1+\frac{x}{\sigma}\right)^{-\alpha},
$$

where $\sigma$ is a scale parameter and $\alpha$ is the shape parameter we are interested in. Since $X$ and $X_{(n)}$ belong to the same domain of attraction of a max-stable distribution (in this case the Fréchet distribution), then the value of $\alpha$ ought not to depend of $n$, and we have departed from the custom of taking $n=1$ and instead have applied the method with $n=278$. This departure from the usual way of applying the threshold approach was motivated by the instability of the results obtained when taking $n=1$. In Subsection 4.6 we will suggest a reason for the instability of the estimation of parameters when taking $n=1$.

We applied the threshold approach with a block size given by $n=278$ and with $u=9 \times 10^{-5}$ as the chosen threshold. With these values for $n$ and $u$, we obtained a set of excesses of size 153. Then we used the maximum likelihood method for the estimation of the parameters in the generalized Pareto distribution given by Equation [5. For these parameters we obtained $\hat{\sigma}=7.59 \times 10^{-4}$ and $\hat{\alpha}=3.34$. For the null hypothesis stating that the 153 excesses come from a Pareto distribution given by Equation 5 , the Pearson chi squared statistic has a $p$-value of 0.875 . In Table 3 we report a $95 \%$ confidence interval for the parameter $\alpha$. This confidence interval was computed by the profile likelihood method.

\subsection{Combining a log-logistic and a Pareto parts}

In Figure 2 we present the log-log plot of the 1000 most extreme values in the data set under study. In the horizontal axis of this Figure 2 it is plotted the quantity $\log x$, and in the vertical axis the quantity $\log \mathrm{P}\{X>x\}$, where $X$ represents an observed solar flare intensity in our data set. In this figure, the points corresponding to intensities $X$ lying in the interval $\left(9 \times 10^{-5}, 8.5 \times 10^{-4}\right)$ are plotted in blue color. The number of solar flare intensities $X$ lying in this interval equals 530. Drawn in red color, in Figure 2 we can see the straight line fitted by least squares to the color blue points. The slope and intercept of the fitted line equal -1.2689 and -16.772 respectively. Henceforth, on a first stance, one might expect that when $x$ is large (larger than $9 \times 10^{-5} \mathrm{~W} \mathrm{~m}^{-2}$ ), then we have the estimate

$$
\mathrm{P}\{X>x\}=\frac{5.1991 \times 10^{-8}}{x^{1.2689}} .
$$

Also in Figure 2, we have plotted in green color the points corresponding to the 28 largest values of $X$, that is to say, those points for which $X>8.5 \times 10^{-4} \mathrm{~W}$ 
$\mathrm{m}^{-2}$. Drawn in orange color, in Figure 2 we can see the straight line that best fits the color green points. The slope and intercept of the fitted line equal -3.0382 and -29.147 respectively. Thus, when $x$ is large (larger than $8.5 \times 10^{-4}$ ), then we have

$$
\mathrm{P}\{X>x\}=\frac{2.1954 \times 10^{-13}}{x^{3.0382}} .
$$

The question now arises as to whether the difference in the two exponents of $x$ in Equations 6 and 7 is due to a pure random fluctuation or whether there is a physical cause that explains this difference. Now let us consider the null hypothesis $\mathrm{H}_{0}$ stating that the difference in the exponents of $x$ in equations 6 and 7 is explained as a random fluctuation. In order to test $\mathrm{H}_{0}$, we produced a total of $10^{4}$ synthetic samples, each of size 77370 , from a population with loglogistic distribution with parameters $\alpha=1.27$ and $\sigma=1.7 \times 10^{-6}$. Here, the value of $\alpha$ was chosen to be approximately the same as in Equation 6 and the value of $\sigma$ was selected by maximum likelihood form a sub-sample of size 1000 of the solar flare intensities data set. A comparison of Equations 2 and 6 makes it clear that the tail of a log-logistic distribution will have a tail as depicted by Equation 6 . See Figure 3 where we present the histogram depicting the distribution the slope of the fitted straight line to the 28 most extreme values in each of the above $10^{4}$ synthetic samples. From these synthetic samples, we estimated the probability that the 28 most extreme values in one of these samples have a distribution as given in Equation 7 with an exponent $\alpha$ larger in absolute value than or equal to 3.0382 . This probability is approximately $4 \times 10^{-5}$. These Monte Carlo computations lead us to reject $\mathrm{H}_{0}$, that is to say, we conclude that the difference in the two exponents of $x$ in Equations 6 and 7 is not explained as a result of a pure random fluctuation.

The rejection of $\mathrm{H}_{0}$ suggests that an attenuation phenomenon is taking place that results in that the most extreme solar flares (those larger than $8.5 \times 10^{-4}$ ) have a lesser probability of occurrence than what is predicted from the model given by Equation 6 .

In view of the former considerations we now propose to model the data set under study by combining a log-logistic distribution and a Pareto distribution. In order to state with precision this idea, let us consider two probability distributions $F_{1}$ and $F_{2}$. We write $\bar{F}(x)=1-F(x)$ for the complementary cumulative distribution function. Let us say that $G$ is the composition at $\sigma_{2}$ of $F_{1}$ and $F_{2}$, if

$$
G(x)= \begin{cases}F_{1}(x) & \text { if } x \leq \sigma_{2} \\ F_{1}\left(\sigma_{2}\right)+\bar{F}_{1}\left(\sigma_{2}\right) F_{2}(x) & \text { if } x>\sigma_{2}\end{cases}
$$

Notice that if $x>\sigma_{2}$ then $\bar{G}(x)=\bar{F}_{1}\left(\sigma_{2}\right) \bar{F}_{2}(x)$. Notice also that if $G(x)$ is defined as in Equation 8 then it is continuous at $x=\sigma_{2}$ and $G(x) \rightarrow 1$ as $x \rightarrow \infty$. This last condition must be satisfied by every cumulative probability distribution function. In the special case that $F_{2}$ is a Pareto distribution of the form

$$
F_{2}(x)=1-\left(\frac{x}{\sigma_{2}}\right)^{-\alpha_{2}}
$$


for $x \geq \sigma_{2}$, then the representation of $G$ in $\log$-log scale is given by

$$
\log \bar{G}\left(e^{x}\right)= \begin{cases}\log \overline{F_{1}}\left(e^{x}\right) & \text { if } x \leq \sigma_{2}, \\ -\alpha_{2} x+\alpha_{2} \log \sigma_{2}+\log \overline{F_{1}}\left(e^{\sigma_{2}}\right) & \text { if } x>\sigma_{2} .\end{cases}
$$

Now we let $F_{1}$ be a log-logistic distribution with parameters $\alpha_{1}$ and $\sigma_{1}$ and $\bar{F}_{2}(x)=\left(\sigma_{2} / x\right)^{\alpha_{2}}$ the Pareto distribution with parameters $\alpha_{2}$ and $\sigma_{2}$. We let $G_{1}$ be the composition at $\sigma_{2}$ of these $F_{1}$ and $F_{2}$. We propose to model the data set under study by this distribution $G_{1}$.

The distribution $G_{1}$ is determined by its four parameters $\alpha_{1}, \sigma_{1}, \alpha_{2}$ and $\sigma_{2}$. For the estimation of these parameters, we divided the data set into two subsets $S_{1}$ and $S_{2}$. In $S_{1}$ we included all data points of magnitude less than or equal to $\sigma_{2}$, where $\sigma_{2}$ is assumed to be known. In $S_{2}$ we included all data points of magnitude greater than $\sigma_{2}$. Given a numerical value $\hat{\sigma}_{2}$ for $\sigma_{2}$, the first two parameters $\alpha_{1}$ and $\sigma_{1}$ where estimated by the method of moments from the data points in $S_{1}$. Here we are assuming that $S_{1}$ is a sample from a log-logistic distribution truncated at $\sigma_{2}$. With the same given value for $\sigma_{2}$, the value of $\alpha_{2}$ was estimated by the maximum likelihood method. Once we have numerical values for $\hat{\alpha}_{1}, \hat{\sigma}_{1}, \hat{\alpha}_{2}$ and $\hat{\sigma}_{2}$, the average distance $\bar{D}\left(\hat{\sigma}_{2}\right)$ from the distribution $\hat{G}_{1}$ to the empirical distribution was computed. By letting $\hat{\sigma}_{2}$ assume a whole range of values, we found that $\bar{D}\left(\hat{\sigma}_{2}\right)$ assumes a minimum value of 0.0139 when we set $\hat{\sigma}_{2}=9 \times 10^{-4}$. It is interesting to note that with the above optimal value for $\hat{\alpha}_{2}$ the set $S_{2}$ contains 28 points. This vindicates the more naive approach for the determination of the stochastic behavior of the few most extreme values in our data set presented at the beginning of this subsection.

Given this optimal value $\hat{\sigma}_{2}=9 \times 10^{-4}$, the rest of the parameters of $G_{1}$ were determined as stated above. For the first parameter we found that $\hat{\alpha}_{1}=1.2409$ with $(1.19,1.30)$ as $95 \%$ confidence interval. For the second parameter we found that $\hat{\sigma}_{1}=0.0160$ with $(0.015,0.017)$ as $95 \%$ confidence interval. For the third parameter of $G_{1}$ we found that $\hat{\alpha}_{2}=3.04$ with $(2.29,4.33)$ as $95 \%$ confidence interval. All these are bootstrap confidence intervals computed by re-sampling the original data set $10^{4}$ times.

It is interesting to compare the upper limit of the above confidence interval for $\alpha_{1}$ with the lower limit of the confidence interval for $\alpha_{2}$. The difference of these is $2.29-1.30=0.99$. This positive difference is in agreement with the rejection of $\mathrm{H}_{0}$ above, that is to say, the rejection of the claim that the few (about 28) most extreme values in our data set follow a probability law with a tail behavior as depicted in Equation 6 .

In Figure 4 we present the log-log plot of the solar flare intensities together with the log-log plot of the complementary distribution function $\bar{G}_{1}(x)$, where $G_{1}(x)$ is as just determined (we omit the hat symbol).

\subsection{A log-logistic part and a tempered Pareto part}

Although with the composition of a log-logistic and a Pareto distributions we have achieved a reasonably good fit to the data under study, it should be remarked that the distribution $G_{1}$ considered in Subsection 4.4 is not without 
inconveniences. Indeed, $G_{1}$ is not differentiable at $\sigma_{2}$ and it is questionable that a non differentiable distribution is to be acceptable as a model for our data set. On the other hand, the number of data points explained by the Pareto part of $G_{1}$ is only 28 , and this is a small number. For the purpose of the estimation of parameters of a proposed model, it is desirable to work with as large a sample as it is possible. In this subsection we consider as a model for our data set a composition of a log-logistic distribution and a tempered Pareto distribution given by

$$
\bar{T}(x)=c x^{-\alpha_{2}} e^{-\beta x}
$$

when $x>\sigma_{2}$ and $\bar{T}(x)=0$ when $x \leq \sigma_{2}$. Here $c=\sigma_{2}^{\alpha_{2}} e^{\beta \sigma_{2}}$ is the corresponding normalizing constant and $\sigma_{2}, \alpha_{2}$ and $\beta$ are parameters of the tempered Pareto distribution. See Meerschaert, Roy, and Shao (2012) and Clauset, Shalizi, and Newman (2009) for information about the tempered Pareto distribution. Now we consider the probability distribution function $G_{2}$ that results from the composition at $\sigma_{2}$ of a log-logistic distribution with parameters $\alpha_{1}, \sigma_{1}$ (Equation 2) and a tempered Pareto distribution with parameters $\alpha_{1}, \beta, \sigma_{2}$ (Equation 10).

For the estimation of the five parameters of $G_{2}$, we proceeded as with the estimation of the four parameters of $G_{1}$. Thus, we considered a set $S_{1}$ containing all data points less than or equal to $\sigma_{2}$ and a set $S_{2}$ containing all data points greater than $\sigma_{2}$. Given a tentative numerical value $\hat{\sigma}_{2}$ for $\sigma_{2}$, we used the method of moments in order to obtain estimates $\hat{\alpha}_{1}$ and $\hat{\sigma}_{1}$, determining the log-logistic part of $G_{2}$, from the set $S_{1}$. With this same numerical value $\hat{\sigma}_{2}$, we used the data points in $S_{2}$ and the method of moments in order to obtain estimates $\hat{\alpha}_{2}$ and $\hat{\beta}$ for the remaining two parameters of the tempered Pareto part of $G_{2}$.

The average distance $\bar{D}\left(\hat{\sigma}_{2}\right)$ from the estimated distribution $\hat{G}_{2}$ and the empirical distribution was computed for a range of numerical values $\hat{\sigma}_{2}$. We found that $\bar{D}\left(\hat{\sigma}_{2}\right)=0.00777$ is the smallest value for this distance, and this smallest value is achieved when $\hat{\sigma}_{2}=8 \times 10^{-6}$. With this optimal value for $\hat{\sigma}_{2}$ we found the estimates for the remaining parameters of $G_{2}$. For the first parameter we found that $\hat{\alpha}_{1}=1.358$ with $(1.338,1.377)$ as $95 \%$ confidence interval. For the second parameter we found that $\hat{\sigma}_{1}=0.0172$ with $(0.0169,0.0174)$ as $95 \%$ confidence interval. For the third parameter of $G_{2}$ we found that $\hat{\alpha}_{2}=1.106$ with (1.074, 1.136) as $95 \%$ confidence interval. For the fourth parameter of $G_{2}$ we found that $\hat{\beta}=0.098$ with $(0.073,0.138)$ as $95 \%$ confidence interval. All the these are bootstrap confidence intervals.

In Figure 5 we present the log-log plot of the solar flare intensities together with the log-log plot of the complementary distribution function $\bar{G}_{2}(x)$, where $G_{2}(x)$ is as just determined (with the hat symbol omitted).

\subsection{Performance of the estimation procedures}

In this subsection we reflect on the consequences for the estimation of the parameter $\alpha$ by applying the block and threshold methods to a sample that presents the same dual tail behaviour as does the data set under study, and which was put in evidence in Subsection 4.4 
When applying the block approach to the estimation of $\alpha$, a set $S_{59}$ of 59 maxima was formed and then it was used for the estimation by maximum likelihood of the parameters of the $\mathrm{G}_{\mathrm{ev}}$ distribution given by Equation 4 , where $\alpha$ is given by $1 / \xi$. If the number of blocks is large (larger than 28 ), then it is highly likely that this sample $S_{59}$ comes from a distribution that results from a mixture of the two distributions given by Equation 7 and a truncated version of the distribution determined by Equation 目. However, the fact that this sample $S_{59}$ comes from a mixture of distinct distributions is not taken into account by the estimation procedure of the parameters of the $\mathrm{G}_{\mathrm{ev}}$ distribution. For example, when considering synthetic samples from the distribution $G_{1}$ considered in Subsection 4.4 where $G_{1}$ is composed of two distributions (log-logistic and Pareto) having tail indices $\alpha_{1}=1.27$ and $\alpha_{2}=3$, then the estimate $\hat{\alpha}$ in the block approach will assume a value in between $\alpha_{1}$ and $\alpha_{2}$, unless the number of blocks is small, in which case $\hat{\alpha}$ will be closer to $\alpha_{2}$, but in this case the estimation errors will be large.

On the other hand, when applying the threshold approach to the solar flare data set, we have a set $S_{28}$ of about 28 data points to which a straight line with a slope about -3 can be fitted. From this sample $S_{28}$ we are to estimate the parameters of the distribution $H$ given in Equation 5. Yet, it is desirable to perform the estimation procedure from a sample $S^{*}$ as large as possible. One can obtain a larger sample $S^{*}$ by choosing a lower threshold $u$. However, if we choose a low enough threshold $u$, then our sample $S^{*}$ will come from a mixture of two Pareto distributions, where one of them has been truncated. When plotted on log-log scale, this sample $S^{*}$ will align itself along two straight line segments that meet in a corner, drawn in gray color in parts (a) and (b) of Figure 6. In this Figure 6 we have also drawn in red color the function

$$
\log H\left(e^{x}\right)=-\alpha x+\alpha \log \sigma-\alpha \log \left(1+\frac{\sigma}{e^{x}}\right),
$$

which is the representation in $\log -\log$ scale of the distribution $H$ given in Equation 5. In this figure, the function $\log H\left(e^{x}\right)$ has been drawn so that it reproduces the two slopes of the gray broken line at the two points drawn in blue color. Notice that when these two blue points are sufficiently close together, then the function $\log H\left(e^{x}\right)$ has a large curvature that results in an overestimation of the parameter $\alpha$, which in the figure corresponds to the absolute value of the slope of the right hand gray straight line segment.

\section{Return periods and expected frequencies}

In this section we report the return levels corresponding to distinct time periods which result from the block, threshold, log - logistic/Pareto and log - logistic/tempered Pareto approaches to the estimation of the tail behavior of the solar flare intensities data set.

\footnotetext{
${ }^{3}$ In terms of Figure 4 the set $S_{59}$ would be a combination of data points on the left and on
} the right of the dashed vertical line in the figure. 
In Table 4 we display the return levels that correspond to distinct return periods computed by the block approach. For example, for a return period of 10 years, we expect to observe once, an event of magnitude $1.68 \times 10^{-3}\left(\mathrm{~W} \mathrm{~m}^{-2}\right)$ or larger. In the third and fourth columns of this table, we report the lower and upper bounds of a $95 \%$ confidence interval for the return level. These confidence intervals were computed by the normal approximation method.

In Table 5 we present return levels together with their $95 \%$ confidence intervals that correspond to distinct return periods computed by the threshold approach. These confidence intervals were also computed by assuming that we have a normal approximation for the distribution of the estimated parameters of the model.

In Table 6 we present the return levels together with their $95 \%$ confidence intervals that correspond to distinct return periods computed from the model which results from the composition of a log-logistic and a Pareto distributions as discussed in Subsection 4.4. In this case, the confidence intervals were computed by the bootstrap method by resampling the original data set $10^{4}$ times.

In Table 7 we present the return levels together with their $95 \%$ confidence intervals corresponding to distinct return periods computed from the model which results from the composition of a log-logistic and a tempered Pareto distributions as discussed in subsection 4.5. These confidence intervals were also computed by the bootstrap method.

From Tables 4 to 7 it is evident that the return levels corresponding to distinct periods are larger when computed from the block and threshold approaches than when they are computed form the log-logistic/Pareto and log-logistic/tempered Pareto models. It is reasonable to contend that this is so because of the inability of the first two approaches to properly deal with a sample that results from a mixture of two distributions with two distinct tails, one heavier than the other. It is quite evident that, in the block approach, the inclusion of data points (in the set $S_{59}$ ) from the first part of the tail of our data set (a tail with $\alpha=1.35$ according to the first estimate in Table 3) results in an underestimation of $\alpha$, which in turn results in an overestimation of the return levels. For the threshold approach, it is more difficult to present clear and convincing reasons in order to support our contend that it also overestimates the return levels. Let it be enough to remark that the data set under study only allowed us to apply the threshold method for estimating $\alpha$ with a rather small set of data points from which we estimated the parameters of distribution $H$ in Equation 5 . Therefore, the threshold method as applied here, does not allow us to describe a long tail (over at least two orders of magnitude) as in the case of a distribution without a dual behavior of its tail (see Stumpf and Porter (2012)).

By performing numerous Monte Carlo simulations with synthetic samples resembling the original data set under study 4 , it became clear that the methods of the extreme value theory use the available information (in the sets $S_{59}$ and $S^{*}$ of Subsection 4.6) more efficiently than the use made of the information (the whole data set) available to the more naive approaches we followed by using

${ }^{4}$ For the generation of these synthetic samples, we used both, $G_{1}$ and $G_{2}$ as the parent distribution. 
compositions of a log-logistic and Pareto parts. It turns out that the information available to the composition models is so large (recall that the size of the entire data set is 77370) that it outweighs the advantage of using the methods of the extreme value theory. The advantage in the amount of information for the composition models over the amount of information in the block and threshold approaches is put into evidence when considering the length of the confidence intervals for $\alpha$ reported in Table 3 Indeed, we have shorter confidence intervals in the composition models than in the block and threshold approaches.

In Table 8 we report the expected number of events of distinct magnitude to be observed in one solar cycle (11 years) computed from the models which result from the composition of a log-logistic and a Pareto distributions and the composition of a log-logistic and tempered Pareto distributions. The expected number of occurrences of the event $\{X>x\}$ in a time period of $n$ years is equal to $\mathrm{E}(Y)$, where $Y$ is a binomial random variable with probability of successes $p=\mathrm{P}\{X>x\}$ and number of trials $\tilde{n}=365.24 n d$, where $d=5.05248$ is the average number of flares observed per day. Notice that this expected number of observations $\mathrm{E}(Y)$ is a function of $d$, which is the number of events per day, and recall that $d$ is not constant over time. In this Table 8 , we also display the corresponding expected number of events reported in the NOAA web pag $₫$. We find a reasonable good agreement between the web page results and the results we obtained form the two composition models. The large amount of information available for the two composition models also results in small confidence intervals for the return levels in Tables 6 and 7

\section{Conclusions}

The aim of this work at the beginning was to determine the tail behavior of the probability distribution of the intensity $X$ of solar flares, as depicted by the parameter $\alpha$ in Equation 1 However, we latter found that the data set under study presents a dual tail behavior. Indeed, we found that the very most extreme values in our data set are less intense than what one would expect on account of the behavior of the rest of the data points. In terms of Figure 4 , the points on the right of the dashed vertical line behave differently from what one would expect from the behavior of the points on the left of the dashed vertical line. This dual tail behavior was confirmed by a hypothesis test in Section 4.4. Now, it is natural to pose the question of whether the attenuation of the intensities is a natural phenomenon pertaining to the physics of the solar activity or whether it is due to a threshold of the measuring instruments (Chamberlin et al., 2009).

It should be noted that the dual behavior, or deviation from a pure power law (as in Equation 1), of the tail of the distribution of the solar flare intensities has already been noticed in the literature (see Wheatland (2010) and Aschwanden and Freeland (2012)). In particular Wheatland (2010) applied Bayesian methods in order to study flare intensities in a single active region, and found evidence for

\footnotetext{
${ }^{5}$ www.swpc.noaa.gov/noaa-scales-explanation
} 
departure from a pure power law behavior. Our findings are in agreement with Wheatland's. The deviation from a pure power law behavior in empirical data has been observed, not only in studies pertaining solar activity, but in other fields of scientific inquiry as well (see Chinnery and North (1975), for example). As evidence mounts in favour to the claim that pure power laws are not completely suitable for describing extreme events in natural phenomena, alternative models appear in the literature (see Chakrabarty and Samorodnitsky (2012); Grabchak (2016)) as modelling resources whose usefulness ought to be explored in the future.

Our results show that on average we can expect one solar flare greater than $\mathrm{X} 23$ each 25 years, that is to say, one such event each two solar cycles. The threshold of $\tilde{X} 20$ is important because the energy level of saturation of the GOES spacecraft is X17.1 (Chamberlin et al., 2009). After the flux of solar flares reaches X20 the instruments do not provide any more data. The peak of energy of the solar flare in progress remains unknown until forensic techniques allow us to determine the intensity of the flare (Kane, McTiernan, and Hurley (2005)). However, it is mandatory to know the energy released in order to start the protocols of civil protection for Space Weather. Fortunately, events like Carrington's (Cliver and Dietrich, 2013) seem not to be very frequent (X40). Our results show that this kind of extreme events have a return period between 131 years (Log-Logistic/Pareto) and 238 years (Log-logistic/Tempered Pareto).

Acknowledgments The authors thank projects for Catedras Conacyt (Conacyt Fellow), Repositorios Institucionales (268273) and Ciencia Basica (254497).

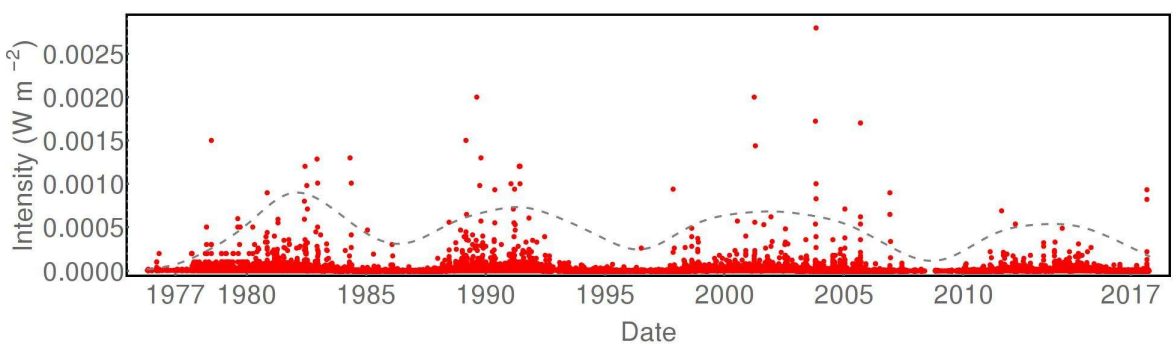

Figure 1. The raw data. The dashed gray line represent the density of event occurrence. 


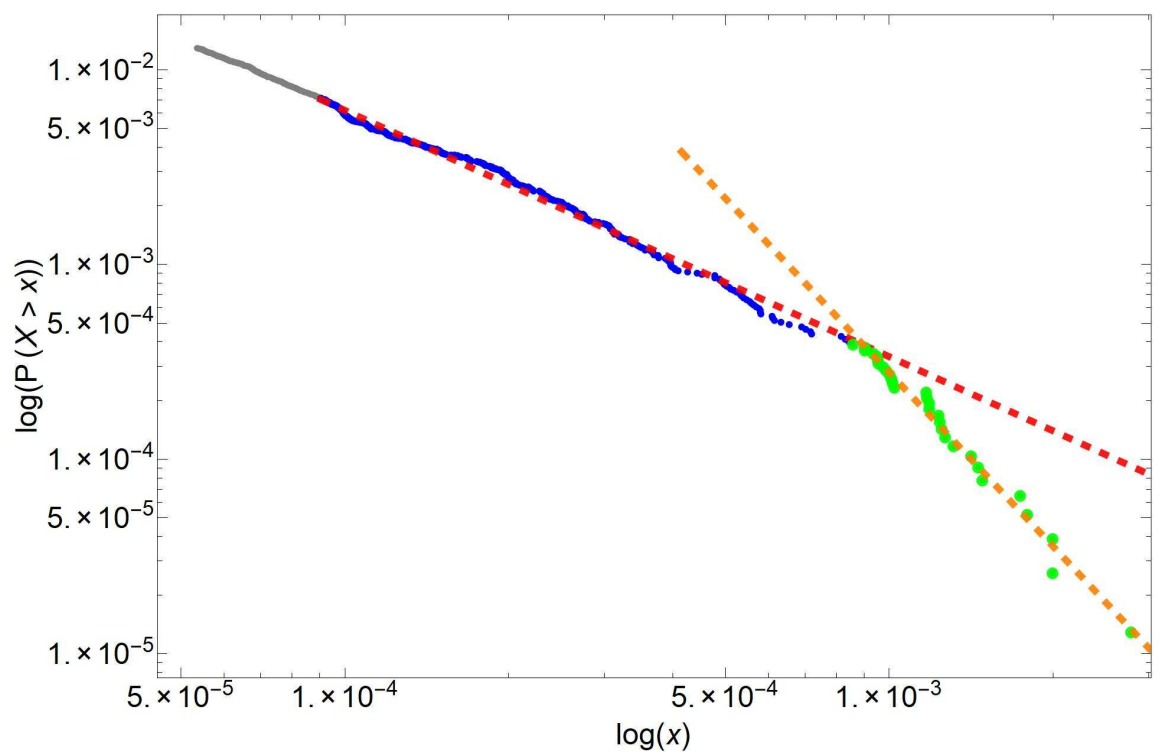

Figure 2. The log-log plot for the 1000 most extreme points in the data set. This figure shows that a change in the slope of the fitted straight line segments is taking place as we move from left to right.

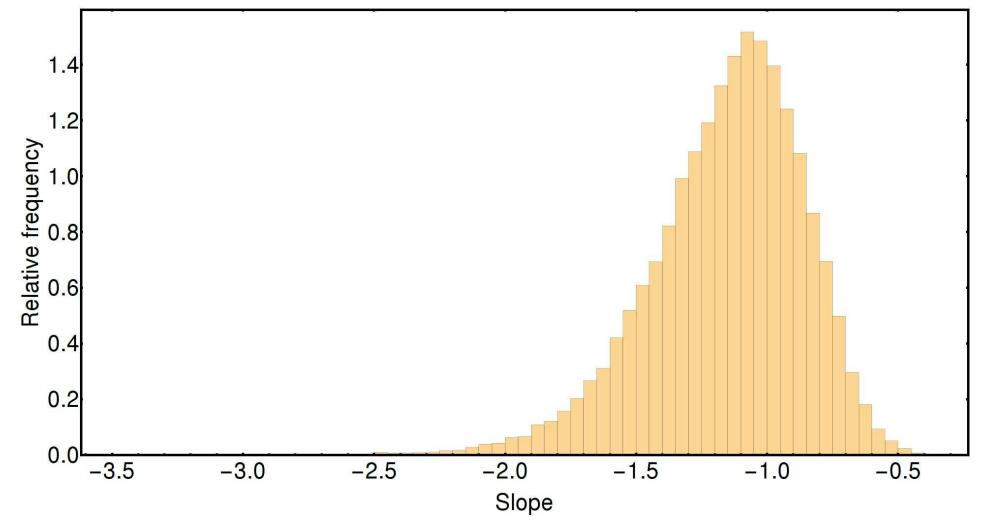

Figure 3. The distribution of the slope of the fitted regression line to the set of the 28 top extremes in $10^{4}$ synthetic samples from a log-logistic distribution with $\alpha=1.27$. From this histogram, it follows that the event $\{\hat{\alpha}>3\}$ has a small probability of occurrence. Here $\hat{\alpha}$ is computed from the 28 most extreme data points in a sample. 


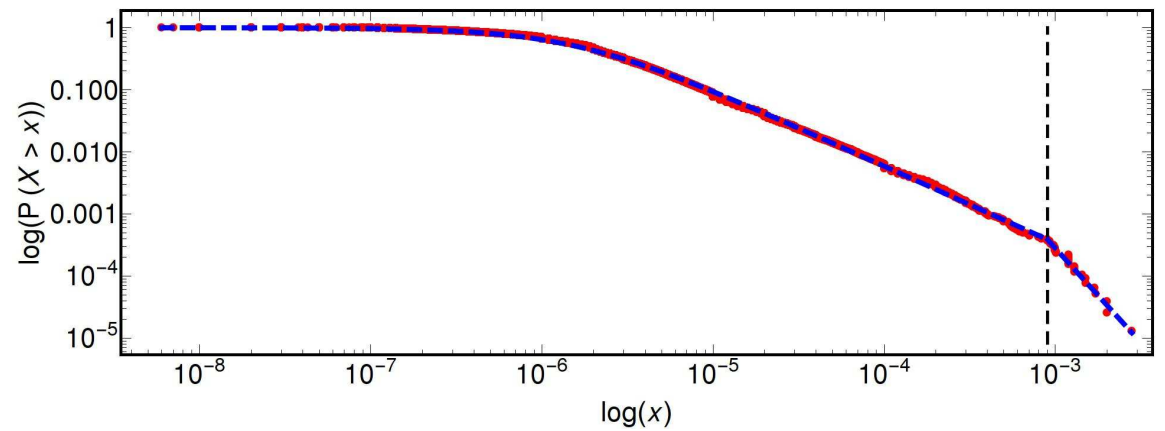

Figure 4. The log-log plot for the full data set and of $\bar{G}_{1}(x)$. To the left of the dashed vertical line, the log-logistic distribution holds. To the right of the dashed line the Pareto distribution holds.

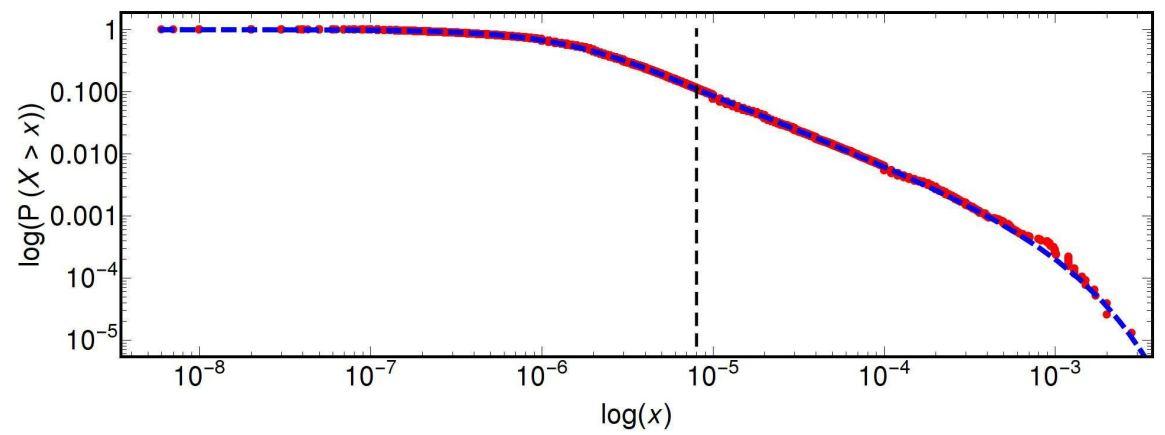

Figure 5. The log-log plot for the full data set and of $\bar{G}_{2}(x)$. To the left of the dashed vertical line, the log-logistic distribution holds. To the right of the dashed line the tempered Pareto distribution holds.
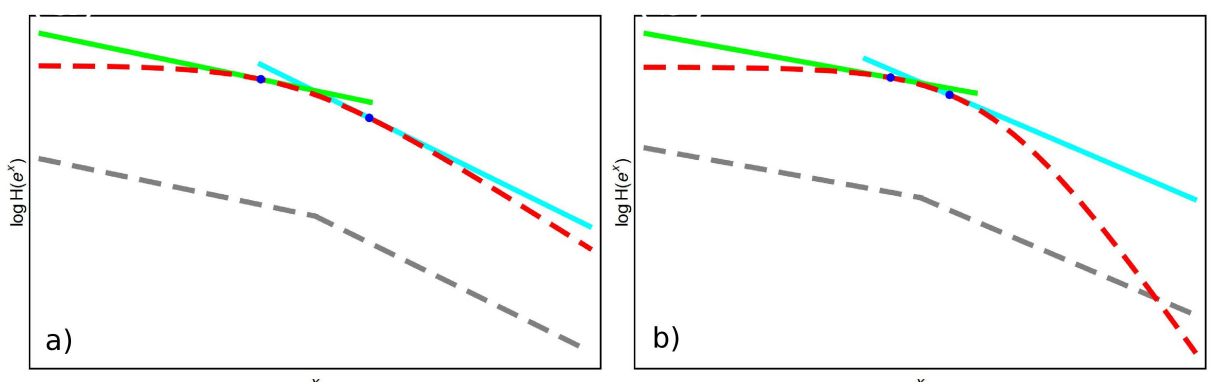

Figure 6. Large curvature as misbehavior in the threshold approach. 


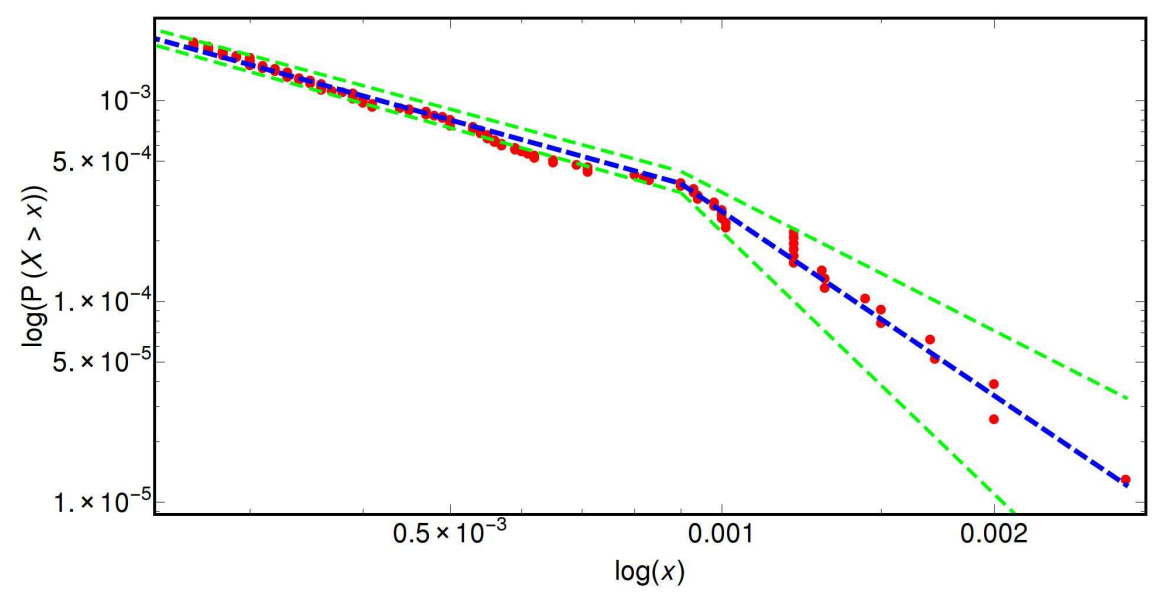

Figure 7. This is the log-log plot of the 150 most extreme points in the data set. The blue dashed line stands for the $\bar{G}_{1}$ complementary distribution function considered in Subsection 4.4 The right hand part of the tails of the two dashed green lines have slopes -4.33 and -2.29 . These slopes correspond to the $95 \%$ confidence interval bounds reported for the parameter $\alpha$ in the second part of the log-logistic/Pareto model in Table 3

Table 1. The distribution of intensities in $1 \times 10^{-4} \mathrm{~W} \mathrm{~m}^{-2}$ of the solar flares.

\begin{tabular}{|c|c|r|r|r|r|}
\hline No. & Range & Data & No. & Range & Data \\
\hline 1 & $<0.05(\mathrm{C} 5)$ & $62364 \mid$ & 11 & $8(\mathrm{X} 8)$ to $10(\mathrm{X} 10)$ & 11 \\
\hline 2 & $0.05(\mathrm{C} 5)$ to $0.1(\mathrm{M} 1)$ & 8162 & 12 & $10(\mathrm{X} 10)$ to $12(\mathrm{X} 12)$ & 5 \\
\hline 3 & $0.1(\mathrm{M} 1)$ to $0.2(\mathrm{M} 2)$ & 3635 & 13 & $12(\mathrm{X} 12)$ to $14(\mathrm{X} 14)$ & 9 \\
\hline 4 & $0.2(\mathrm{M} 2)$ to $0.3(\mathrm{M} 3)$ & 1221 & 14 & $14(\mathrm{X} 14)$ to $16(\mathrm{X} 16)$ & 3 \\
\hline 5 & $0.3(\mathrm{M} 3)$ to $0.5(\mathrm{M} 5)$ & $881 \mid$ & 15 & $16(\mathrm{X} 16)$ to $18(\mathrm{X} 18)$ & 2 \\
\hline 6 & $0.5(\mathrm{M} 5)$ to $1(\mathrm{X} 1)$ & 615 & 16 & $18(\mathrm{X} 18)$ to $20(\mathrm{X} 20)$ & 0 \\
\hline 7 & $1(\mathrm{X} 1)$ to $2(\mathrm{X} 2)$ & $263 \mid$ & 17 & $20(\mathrm{X} 20)$ to $22(\mathrm{X} 22)$ & 2 \\
\hline 8 & $2(\mathrm{X} 2)$ to $4(\mathrm{X} 4)$ & 151 & 18 & $22(\mathrm{X} 22)$ to $24(\mathrm{X} 24)$ & 0 \\
\hline 9 & $4(\mathrm{X} 4)$ to $6(\mathrm{X} 6)$ & 35 & 19 & $24(\mathrm{X} 24)$ to $26(\mathrm{X} 26)$ & 0 \\
\hline 10 & $6(\mathrm{X} 6)$ to $8(\mathrm{X} 8)$ & 10 & 20 & $26(\mathrm{X} 26)$ to $28(\mathrm{X} 28)$ & 1 \\
\hline
\end{tabular}


Table 2. The list of the most intense events in the data set (magnitude in $1 \times 10^{-4} \mathrm{~W} \mathrm{~m}^{-2}$ ).

\begin{tabular}{|c|c|c|c|c|c|}
\hline No. & Magnitude & Date & No. & Magnitude & Date \\
\hline 1 & 28 (X28) & 4 Nov 2003 & 16 & $12(\mathrm{X} 12)$ & 6 Jun 1982 \\
\hline 2 & $20(\mathrm{X} 20)$ & 2 Apr 2001 & 17 & $12(\mathrm{X} 12)$ & 1 Jun 1982 \\
\hline 3 & $20(\mathrm{X} 20)$ & 16 Aug 1989 & 18 & $10(\mathrm{X} 10)$ & 20 May 1984 \\
\hline 4 & 17 (X17) & 28 Oct 2003 & 19 & 10 (X10) & 17 Dec 1982 \\
\hline 5 & 17 (X17) & 7 Sept 2005 & 20 & $10(\mathrm{X} 10)$ & 20 Oct 2003 \\
\hline 6 & $15(\mathrm{X} 15)$ & 6 Mar 1989 & 21 & $10(\mathrm{X} 10)$ & 9 Jun 1991 \\
\hline 7 & 15 (X15) & 11 Jul 1978 & 22 & 10 (X10) & 25 Jan 1991 \\
\hline 8 & 14 (X14) & 15 Apr 2001 & 23 & $10(\mathrm{X} 10)$ & 29 Sept 1989 \\
\hline 9 & 13 (X13) & 19 Oct 1989 & 24 & 10 (X10) & 9 Jul 1982 \\
\hline 10 & 13 (X13) & 25 Apr 1984 & 25 & 9 (X9) & 6 Nov 1997 \\
\hline 11 & 13 (X13) & 15 Dec 1982 & 26 & 9 (X9) & 22 Mar 1991 \\
\hline 12 & 12 (X12) & 15 Jun 1991 & 27 & 9 (X9) & 6 Sept 2017 \\
\hline 13 & $12(\mathrm{X} 12)$ & 11 Jun 1991 & 28 & $9(\mathrm{X} 9)$ & 24 May 1990 \\
\hline 14 & 12 (X12) & 6 Jun 1991 & 29 & 9 (X9) & 5 Dec 2006 \\
\hline 15 & $12(\mathrm{X} 12)$ & 1 Jun 1991 & 30 & 9 (X9) & 6 Nov 1980 \\
\hline
\end{tabular}

Table 3. The estimated parameter $\alpha$ in various models. In the column "Fit" we report the Kolmogorov-Smirnov distance from the empirical distribution to the distribution of the model in turn. For the Pearson chi squared statistic, the null hypothesis that the data fits the model is not rejected for those entries in column " $\alpha$ " marked with an asterisk.

\begin{tabular}{|c|c|c|c|c|}
\hline Model & $\alpha$ & Conf. int. & Sample & Fit \\
\hline Log-logistic & $1.35^{*}$ & $(1.25,1.45)$ & 500 & 0.032 \\
\hline Block & $2.41^{*}$ & $(1.54,5.57)$ & 59 & 0.079 \\
\hline Threshold & $3.34^{*}$ & $(1.83,8.77)$ & 153 & 0.048 \\
\hline Log-logistic/Pareto 1 & 1.24 & $(1.19,1.30)$ & 77342 & 0.055 \\
\hline Log-logistic/Pareto 2 & $3.04^{*}$ & $(2.29,4.33)$ & 28 & $6 \times 10^{-5}$ \\
\hline Log-logistic/tempered Pareto 1 & 1.36 & $(1.34,1.38)$ & 68724 & 0.029 \\
\hline Log-logistic/tempered Pareto 2 & 1.11 & $(1.07,1.14)$ & 8646 & 0.01 \\
\hline
\end{tabular}


Table 4. Return levels $\times 10^{-4}\left(\mathrm{~W} \mathrm{~m}^{-2}\right)$ for the block approach.

\begin{tabular}{|c|c|c|c|}
\hline Period & Return level & Lower bound & Upper bound \\
\hline \hline 10 & 16.80 & 10.26 & 23.34 \\
\hline 25 & 26.49 & 12.39 & 40.58 \\
\hline 50 & 36.61 & 12.69 & 60.53 \\
\hline 100 & 50.07 & 12.69 & 89.24 \\
\hline 150 & 59.92 & 8.32 & 111.5 \\
\hline
\end{tabular}

Table 5. Return levels $\times 10^{-4}\left(\mathrm{~W} \mathrm{~m}^{-2}\right)$ for the threshold approach.

\begin{tabular}{|c|c|c|c|}
\hline Period & Return level & Lower bound & Upper bound \\
\hline 10 & 19.11 & 12.10 & 26.12 \\
\hline 25 & 27.55 & 13.75 & 41.36 \\
\hline 50 & 35.67 & 14.00 & 57.34 \\
\hline 100 & 45.66 & 12.89 & 78.44 \\
\hline 150 & 52.55 & 11.35 & 93.75 \\
\hline
\end{tabular}

Table 6. Return levels $\times 10^{-4}\left(\mathrm{~W} \mathrm{~m}^{-2}\right)$ for the log-logistic/Pareto model.

\begin{tabular}{|c|c|c|c|}
\hline Period & Return level & Lower bound & Upper bound \\
\hline 10 & 17.16 & 13.98 & 21.55 \\
\hline 25 & 23.19 & 17.35 & 32.02 \\
\hline 50 & 29.12 & 20.38 & 43.40 \\
\hline 100 & 36.57 & 23.95 & 58.65 \\
\hline 150 & 41.79 & 26.33 & 70.01 \\
\hline
\end{tabular}


Table 7. Return levels $\times 10^{-4}\left(\mathrm{~W} \mathrm{~m}^{-2}\right)$ for the log-logistic/tempered Pareto model.

\begin{tabular}{|c|c|c|c|}
\hline Period & Return level & Lower bound & Upper bound \\
\hline 10 & 17.19 & 14.04 & 20.37 \\
\hline 25 & 23.17 & 18.51 & 27.94 \\
\hline 50 & 28.07 & 22.15 & 34.27 \\
\hline 100 & 33.24 & 25.95 & 41.00 \\
\hline 150 & 36.36 & 28.21 & 45.04 \\
\hline
\end{tabular}

Table 8. Expected frequency of event occurrence computed by the log-logistic/Pareto model (LL/P) and by the log-logistic/tempered Pareto model (LL/TP).

\begin{tabular}{|c|c|c|c|c|c|}
\hline Intensity $\left(\mathrm{W} / \mathrm{m}^{2}\right)$ & NOAA & LL/P & Conf. Int. & LL/TP & Conf. Int. \\
\hline $10^{-5}(\mathrm{M} 1)$ & 2000 & 1894 & $(1849,1940)$ & 1745 & $(1677,1815)$ \\
\hline $5 \times 10^{-5}(\mathrm{M} 5)$ & 350 & 279 & $(254,305)$ & 283 & $(264,303)$ \\
\hline $10^{-4}(\mathrm{X} 1)$ & 175 & 119 & $(104,135)$ & 125 & $(115,136)$ \\
\hline $10^{-3}(\mathrm{X} 10)$ & 8 & 7.87 & $(6.04,10.04)$ & 4.00 & $(2.77,5.24)$ \\
\hline $2 \times 10^{-3}(\mathrm{X} 20)$ & $<1$ & 4.95 & $(1.56,9.27)$ & 0.70 & $(0.33,1.15)$ \\
\hline
\end{tabular}




\section{References}

Ackermann, M., Ajello, M., Albert, A., Allafort, A., Baldini, L., Barbiellini, G., Bastieri, D., Bechtol, K., Bellazzini, R., Bissaldi, E., Bonamente, E., Bottacini, E., Bouvier, A., Brandt, T.J., Bregeon, J., Brigida, M., Bruel, P., Buehler, R., Buson, S., Caliandro, G.A., Cameron, R.A., Caraveo, P.A., Cecchi, C., Charles, E., Chekhtman, A., Chen, Q., Chiang, J., Chiaro, G., Ciprini, S., Claus, R., Cohen-Tanugi, J., Conrad, J., Cutini, S., D'Ammando, F., de Angelis, A., de Palma, F., Dermer, C.D., Desiante, R., Digel, S.W., Di Venere, L., Silva, E.d.C.e., Drell, P.S., Drlica-Wagner, A., Favuzzi, C., Fegan, S.J., Focke, W.B., Franckowiak, A., Fukazawa, Y., Funk, S., Fusco, P., Gargano, F., Gasparrini, D., Germani, S., Giglietto, N., Giordano, F., Giroletti, M., Glanzman, T., Godfrey, G., Grenier, I.A., Grove, J.E., Guiriec, S., Hadasch, D., Hayashida, M., Hays, E., Horan, D., Hughes, R.E., Inoue, Y., Jackson, M.S., Jogler, T., Jóhannesson, G., Johnson, W.N., Kamae, T., Kawano, T., Knödlseder, J., Kuss, M., Lande, J., Larsson, S., Latronico, L., Lemoine-Goumard, M., Longo, F., Loparco, F., Lott, B., Lovellette, M.N., Lubrano, P., Mayer, M., Mazziotta, M.N., McEnery, J.E., Michelson, P.F., Mizuno, T., Moiseev, A.A., Monte, C., Monzani, M.E., Moretti, E., Morselli, A., Moskalenko, I.V., Murgia, S., Murphy, R., Nemmen, R., Nuss, E., Ohno, M., Ohsugi, T., Okumura, A., Omodei, N., Orienti, M., Orlando, E., Ormes, J.F., Paneque, D., Panetta, J.H., Perkins, J.S., Pesce-Rollins, M., Petrosian, V., Piron, F., Pivato, G., Porter, T.A., Rainò, S., Rando, R., Razzano, M., Reimer, A., Reimer, O., Ritz, S., Schulz, A., Sgrò, C., Siskind, E.J., Spandre, G., Spinelli, P., Takahashi, H., Takeuchi, Y., Tanaka, Y., Thayer, J.G., Thayer, J.B., Thompson, D.J., Tibaldo, L., Tinivella, M., Tosti, G., Troja, E., Tronconi, V., Usher, T.L., Vandenbroucke, J., Vasileiou, V., Vianello, G., Vitale, V., Werner, M., Winer, B.L., Wood, D.L., Wood, K.S., Wood, M., Yang, Z., Fermi LAT Collaboration: 2014, High-energy Gamma-Ray Emission from Solar Flares: Summary of Fermi Large Area Telescope Detections and Analysis of Two M-class Flares. Astrophys. $J .787,15$. DOI ADS [2014ApJ...787...15A]

Aschwanden, M.J., Freeland, S.L.: 2012, Automated solar flare statistics in soft x-rays over 37 years of goes observations: the invariance of self-organized criticality during three solar cycles. The Astrophysical Journal 754(2), 112. [Aschwanden]

Benz, A.O., Güdel, M.: 2010, Physical Processes in Magnetically Driven Flares on the Sun, Stars, and Young Stellar Objects. Annual Review of Astronomy and Astrophysics 48, 241. DOI ADS [2010ARA\&A..48..241B]

Canfield, R.C., de La Beaujardiere, J.-F., Fan, Y., Leka, K.D., McClymont, A.N., Metcalf, T.R., Mickey, D.L., Wuelser, J.-P., Lites, B.W.: 1993, The morphology of flare phenomena, magnetic fields, and electric currents in active regions. I - Introduction and methods. Astrophys. J. 411, 362. DOI ADS] [1993ApJ ...411..362C]

Castillo, E., Hadi, A.S., Balakrishnan, N., Sarabia, J.-M.: 2005, Extreme value and related models with applications in engineering and science. [Castillo]

Chakrabarty, A., Samorodnitsky, G.: 2012, Understanding heavy tails in a bounded world or, is a truncated heavy tail heavy or not? Stochastic Models 28(1), 109. [Chakrabarty]

Chamberlin, P.C., Woods, T.N., Eparvier, F.G., Jones, A.R.: 2009, Next generation X-ray sensor (XRS) for the NOAA GOES-R satellite series. In: Solar Physics and Space Weather Instrumentation III, Proceedings of the SPIE 7438, 743802. [DOI ADS] [goes]

Chertok, I.M., Belov, A.V.: 2017, Long- and Mid-Term Variations of the Soft X-ray Flare Type in Solar Cycles. Solar Phys. 292, 144. DOI ADS. [2017SoPh..292..144C]

Chinnery, M.A., North, R.G.: 1975, The frequency of very large earthquakes. Science 190, 1197. [Chinnery]

Clauset, A., Shalizi, C.R., Newman, M.E.: 2009, Power-law distributions in empirical data. SIAM review 51(4), 661. [Clauset]

Cliver, E.W., Dietrich, W.F.: 2013, The 1859 space weather event revisited: limits of extreme activity. Journal of Space Weather and Space Climate 3(27), A31. DOI ADS [2013JSWSC . . .3A . .31C]

Coles, S.: 2001, An introduction to statistical modeling of extreme values, Springer Series in Statistics, Springer, Bristol UK. [Coles]

de Haan, L., Ferreira, A.: 2007, Extreme value theory: An introduction, Springer Series in Operations Research and Financial Engineering, Springer, New York. ISBN 9780387344713. [deHaan]

Diaconis, P., Efron, B.: 1983, Computer-intensive methods in statistics. Scientific American 248(5), 116. [Diaconis] 
DiCiccio, T.J., Efron, B.: 1996, Bootstrap confidence intervals. Statistical science, 189. [DiCiccio]

Gold, T., Hoyle, F.: 1960, On the origin of solar flares. Mon. Not. Roy. Astron. Soc. 120, 89. DOI ADS [1960MNRAS.120 . .89G]

Grabchak, M.: 2016, Tempered stable distributions. In: Tempered Stable Distributions, Springer, Charlotte, NC, USA. [Grabchak]

Jonas, S., McCarron, E.D.: 2016, White house releases national space weather strategy and action plan. Space Weather 14(2), 54. 2015SW001357. DOI http://dx.doi.org/10.1002/ 2015SW001357. [SWE:SWE20303]

Kane, S.R., McTiernan, J.M., Hurley, K.: 2005, Multispacecraft observations of the hard Xray emission from the giant solar flare on 2003 November 4. Astron. Astrophys. 433, 1133. DOI ADS [2005A\&A...433.1133K]

Katsova, M.M., Kitchatinov, L.L., Livshits, M.A., Moss, D.L., Sokoloff, D.D., Usoskin, I.G.: 2018, Can superflares occur on the sun? a view from dynamo theory. Astronomy Reports 62(1), 72. DOI https://doi.org/10.1134/S106377291801002X. [Katsova2018]

Koons, H.C.: 2001, Statistical analysis of extreme values in space science. J. Geophys. Res. 106, 10915. DOI ADS [Koons]

Leadbetter, M.R., Lindgren, G., Rootzén, H.: 2012, Extremes and related properties of random sequences and processes, Springer, New York. [Leadbetter]

Meerschaert, M.M., Roy, P., Shao, Q.: 2012, Parameter estimation for exponentially tempered power law distributions. Communications in Statistics-Theory and Methods 41(10), 1839. DOI [Meerschaert]

Riley, P.: 2012, On the probability of occurrence of extreme space weather events. Space Weather 10(2). [Riley]

Stumpf, M.P.H., Porter, M.A.: 2012, Critical truths about power laws. Science 335(6069), 665. DOI http://science.sciencemag.org/content/335/6069/665. [Stumpf]

Toriumi, S., Schrijver, C.J., Harra, L.K., Hudson, H., Nagashima, K.: 2017, Magnetic Properties of Solar Active Regions That Govern Large Solar Flares and Eruptions. Astrophys. J. 834, 56. DOI ADS [2017ApJ . . 834 ...56T]

Wheatland, M.S.: 2010, Evidence for Departure from a Power-Law Flare Size Distribution for a Small Solar Active Region. Astrophys. J. 710, 1324. DOI ADS. [Wheatland] 